\title{
ANÁLISIS DE SISMICIDAD ASOCIADA AL LLENADO DE EMBALSES. CASO DEL CENTRO DE PRODUCCIÓN DE PIRRÍS (COSTA RICA)
}

\author{
ANALYSIS OF RESERVOIR TRIGGERED SEISMICITY.CASE OF PIRRIS RESERVOIR (COSTA RICA) \\ Sandra Ruiz-Barajas ${ }^{a^{*}}$, Guillermo E. Alvarado ${ }^{b}$, Belén Benito Oterino ${ }^{a}$, Álvaro Climent ${ }^{\mathrm{b}}$ \\ ${ }^{a}$ Dpto. Ingeniería Topográfica y Cartografía, Universidad Politécnica de Madrid, Crta de Valencia km 7, 28031, Madrid, España. \\ sandra.ruizb@upm.es \\ ${ }^{\mathrm{b}}$ Area de Amenazas y Auscultación Sismológica y Volcánica, Instituto Costarricense de Electricidad, San José, Costa Rica
}

\begin{abstract}
:
The presented work is the result of a new collaboration between the Costa Rican Institute of Electricity (ICE) and the Technical University of Madrid (UPM) for the investigation of induced seismicity issues. Triggered seismicity associated with the filling of artificial water reservoirs is known since six decades ago. However, it is the case of triggered seismicity more complicated to model their effects. More than 100 cases of reservoir induced/triggered seismicity have been collected around the world and this issue continues today being a research topic of great importance. Indeed, new developments and contributions are constantly being made with the aim of achieve a better understanding of their characteristics and genesis.

The main aim of the presented work is to analyze the spatial-temporal evolution of the seismic events recorded around the Pirrís reservoir before, during and after its filled. With the analysis of the seismic events we try to know and control the influence of the reservoir operations on the seismic activity of the area. Different parameters are studied, using methodologies proposed by different authors, in order to explain the possible effects of reservoir filling in changing the stress conditions in the environment and to detect any possible anomaly. Moreover, some analysis have been done in order to find a possible correlation between the water level in the reservoir and the evolution of the seismic activity recorded.
\end{abstract}

Overall, the results of this study will provide important conclusions about the sensitivity of certain parameters to evaluate and model the effects of filling reservoirs on the seismic activity in the vicinity and we will present the spatial-temporal evolution of the seismicity associated with the specific case of the Pirrís hydraulic project.

Key words: Seismicity, Reservoir, Geophysics, Seismic hazard

\section{Resumen:}

El trabajo presentado es el resultado de una colaboración iniciada entre el Instituto Costarricense de Electricidad (ICE) y la Universidad Politécnica de Madrid (UPM) para la investigación de la sismicidad inducida por causas antrópicas. La sismicidad disparada por el llenado de grandes embalses es un hecho conocido desde hace décadas, siendo uno de los tipos de sismicidad más complejos a la hora de modelizar sus efectos. Hasta ahora, más de 100 casos de sismicidad disparada por embalses han sido recogidos en todo el mundo, por lo que el tema de la sismicidad inducida se ha convertido en una línea de investigación cada vez más importante y estudiada.

El objetivo principal de este trabajo se centra en analizar la evolución espacio-temporal de la sismicidad registrada en los alrededores del embalse de Pirrís (Costa Rica) en tres fases: antes, durante y después de su llenado. Con dicho análisis se pretende conocer y controlar la influencia que las operaciones efectuadas en el embalse tienen en la actividad sísmica de la zona. Se han estudiado diferentes parámetros sísmicos y se ha analizado su variación en el tiempo (diferenciando entre las tres fases temporales citadas con el objetivo final de explicar los posibles efectos que el llenado del embalse puede tener en las condiciones de esfuerzos en el entorno. Además, se incluye un primer análisis cualitativo para identificar la posible correlación entre las variaciones del nivel de agua en el embalse y la evolución de la actividad sísmica registrada.

En este trabajo se presenta un detallado análisis de la evolución espacio-temporal de la sismicidad asociada con el caso específico del proyecto hidráulico Pirrís. En general, los resultados presentados (aunque todavía provisionales) proporcionan una idea sobre la sensibilidad de ciertos parámetros para evaluar y modelar los efectos del llenado de embalses sobre la actividad sísmica del entorno.

Palabras clave: Sismicidad, Embalse, Geofísica, Peligrosidad sísmica 


\section{Introducción}

El trabajo que se presenta es el resultado de una colaboración iniciada entre el Instituto Costarricense de Electricidad (ICE) y la Universidad Politécnica de Madrid (UPM) para la investigación de la sismicidad inducida por causas antrópicas. La ocurrencia de sismos asociados a ciertas actividades humanas, es un hecho observado y reconocido desde hace décadas. No obstante, los casos de sismicidad inducida se han incrementado en los últimos años debido al aumento de proyectos en los que se actúa sobre el medio natural, alterando su estado de esfuerzos.

Diferentes actividades humanas pueden desencadenar la ocurrencia de sismos: explotación de minas, extracción de hidrocarburos convencionales o no convencionales, almacenamiento subterráneo de $\mathrm{CO}_{2}$ para evitar su difusión a la atmósfera, sistemas de obtención de energía geotérmica, inyección de gas en el subsuelo para su almacenamiento, llenado de grandes embalses, etc. Son numerosos los adjetivos que se pueden encontrar en la literatura especializada para describir a este tipo de sismicidad asociada a actividades humanas. Algunos de los términos más utilizados son: sismicidad man-made, inducida, artificial, disparada, anticipada, etc.

Determinar si la sismicidad de una zona es de tipo natural o ha podido ser inducida por la modificación de los estados de esfuerzos del medio por alguna actividad antrópica en la región, no es una tarea fácil. Un factor que complica la identificación de la naturaleza de un terremoto es la dificultad de discernir su pertenencia a una serie sísmica.

Se entiende por sismicidad propiamente natural aquella que se asocia a la energía liberada por las fallas preexistentes en un área, orientadas de acuerdo a la sismotectónica regional y local de una zona.

Cuando se trata de la sismicidad asociada a actividades antrópicas, es crucial diferenciar entre la sismicidad relacionada directamente con la introducción de una energía extra en el sistema, que sería propiamente la sismicidad «inducida», o la sismicidad provocada en una falla próxima o estructura tectónica capaz de generar terremotos, lo que constituiría la sismicidad «disparada o anticipada». En general, cuanta más energía se aporta al sistema, mayor es la sismicidad inducida (número de sismos y tamaño de los mismos), y cuanto más activa es una región, más propensa es a la generación de nuevos eventos tectónicos.

En el caso de la sismicidad inducida propiamente dicha, el cese de la actividad humana de aporte de energía conllevaría el cese de la sismicidad inducida (quizá con un cierto tiempo de retraso). Los sismos inducidos suelen ser de magnitud baja o muy baja (incluso de magnitud negativa).

En el caso de la sismicidad disparada o anticipada, la alteración del estado local de esfuerzos, en especial sobre una falla próxima y cercana a la culminación de su ciclo sísmico (esto es, en un estado críticamente tensionado) puede adelantar la ocurrencia de un sismo que en una situación natural (sin actividad humana de por medio) ocurriría más tarde. Así, un pequeño cambio de esfuerzos (de un bar), podría dar lugar a la generación de un sismo mucho mayor. En este caso la actividad antrópica acelera la ocurrencia de sismos que eventualmente podrían haber ocurrido de forma natural mas tarde.

Algunos autores (McGarr and Simpson 1997) sostienen que la sismicidad asociada con actividades de extracción o inyección de fluidos se clasifica como un tipo de sismicidad inducida mientras que los sismos asociados al llenado o vaciado de embalses serían de naturaleza anticipada (Reservoir Triggered Seismicity, RTS) (ICOLD, International Commission On Large Dams). Este trabajo se centra precisamente en el análisis de éste último tipo de sismicidad. Se presenta un primer análisis espacio-temporal de los eventos sísmicos registrados en los alrededores de una de las obras del Instituto Costarricense de Electricidad (ICE), el Centro de Producción (CP) de Pirrís. Se ha analizado la información sismológica antes, durante y después del llenado del embalse, con el objetivo principal de poder ver la posible afectación que la operación de llenado ha tenido sobre las condiciones sismotectónicas de la zona e identificar la posible ocurrencia de sismicidad disparada en las proximidades. Éste primer análisis ha permitido encontrar diferentes indicadores que evidencian cambios en el patrón de sismicidad antes y después del llenado del embalse.

\section{Sismicidad disparada por embalses (RTS)}

Se entiende por sismicidad disparada por embalses (Reservoir Triggered Seismicity, RTS) la distribución temporal y espacial de eventos sísmicos cuyo origen está asociado con las operaciones de un embalse. Se trata por tanto de una sismicidad que surge de manera anómala durante y después del llenado de un embalse (ICOLD 2004).

Algunas de las características difererenciadoras de este tipo de sismicidad son:

- Los sismos suelen agruparse en clusters alrededor del embalse.

- Los sismos suelen ser muy superficiales. Aunque se ha documentado algún caso de RTS con profundidades de $25 \mathrm{~km}$ (Kebeasy et al. 1987)

- En la mayoría de los casos, la sismicidad empieza poco después del comienzo del llenado y crece conforme aumenta el nivel de agua en el reservorio.

- La mayor parte de eventos disparados identificados hasta ahora, se asocian con fallas de mecanismo normal o de desgarre. Son menos los casos observados de RTS asociados con fallas de tipo inverso.

Además, deben existir ciertas condiciones geológicas e hidrogeológicas especiales para el disparo de sismos en la zona, como es la existencia de fallas activas que se encuentren próximas a un estado de rotura en los alrededores del embalse.

La sismicidad entorno al embalse puede dispararse fundamentalmente por dos causas:

- El propio peso de la masa de agua. Ésto provoca cambios de esfuerzos estáticos en el medio, de forma inmediata tras el llenado del embalse, pues aumenta la presión sobre el fondo del embalse.

- $\quad$ El efecto de la propagación de la presión de poros por el medio. Consiste en el aumento de la presencia de agua en los poros y su difusión por las 
zonas permeables que existan por debajo y en los alrededores del embalse. Ésto produce un cambio drástico en las condiciones hidrológicas de la zona.

Son diferentes las clasificaciones que se pueden encontrar en la literatura y que hacen referencia a la sismicidad asociada a las operaciones en grandes embalses (Talwani 1997b; Simpson et al. 1988; Gupta 2002). Una de las interpretaciones más extendidas (Simpson et al. 1988) sostiene que una respuesta inducida instantáneamente después del llenado (rapid response) es debida principalmente al peso de agua añadida, mientras que una respuesta retardada en el tiempo (Delayed response) está relacionada con una más lenta propagación de la presión de poros.

\subsection{Antecedentes}

La sismicidad disparada por embalses (RTS) es un fenómeno conocido desde la década de 1930 y se ha considerado un factor importante que puede afectar a las condiciones físicas del medio. El primer caso reportado ocurrió en el embalse Mear (EE.UU) creado por la presa Hoover. Sin embargo, no fue hasta la década de los 60 cuando este tema cobró mayor importancia, tras la sismicidad registrada en las proximidades de la presa de Xinfengjiang (China) y Koyna (India), donde se alcanzaron magnitudes superiores a 6.0, produciéndose importantes daños estructurales en ellas y consecuencias graves (Gupta 2002).

Existen otros casos en zonas altamente sísmicas, en los que la sismicidad del entorno ha provocado el fallo de presas e importantes daños estructurales en ellas, como por ejemplo la presa de Sefid Rud (Irán) o la presa de Shih Kang (Taiwan). Por tanto, mantener un monitoreo sismológico en este tipo de estructuras es fundamental para el control de su seguridad. Esta instrumentación permite determinar el comportamiento dinámico de la misma y eventualmente activar alarmas que ayuden a la rápida acción de medidas de emergencia, para evitar efectos y consecuencias sobre la propia estructura y su funcionamiento y, con ello, sobre la población y sus actividades socio-económicas relacionadas.

Dado que los posibles eventos asociados con las operaciones de un embalse suelen ser de magnitud moderada, no suelen suponer un problema para el comportamiento de la presa, pues normalmente estas se diseñan para soportar eventos de mayor magnitud (sobre todo en zonas altamente sísmicas). No obstante, en ciertos casos, la sismicidad inducida puede afectar a los edificios y estructuras situadas en las proximidades de la presa que presentan una mayor vulnerabilidad y con ello un mayor riesgo a tener algún tipo de daño. Por ello, el control de la sismicidad en el entorno es además fundamental para la seguridad y tranquilidad de la población en la zona.

\section{Inclusión de la sismicidad inducida para la evaluación de la peligrosidad sísmica}

El aumento del número de sismos sentidos por la población e inducidos por diferentes causas antrópicas está haciendo que haya cada vez más trabajos centrados en analizar la forma de incluir este tipo de sismicidad en un estudio de peligrosidad sísmica y conocer cual es su repercusión en la estimación del movimiento final esperado.

En un estudio tradicional de peligrosidad PSHA (Probabilistic Seismic Hazard Assessment) se determina la probabilidad de excedencia de un cierto nivel de movimiento para un largo periodo de tiempo. Para ello se parte del catálogo de terremotos históricos e instrumentales y se hace uso de modelos zonificados o de modelos de fallas con sus correspondientes leyes de recurrencia. En este tipo de estudios se determina la peligrosidad o movimiento esperado para plazo de tiempo medio-largo.

Sin embrago, en un estudio probabilista considerando sismicidad inducida PISHA (Probabilistic Induced Seismic Hazard Assessment), lo que se pretende es estimar la peligrosidad a corto plazo (días, horas o meses), pues lo que interesa es el seguimiento de la serie sísmica y sus cambios en función del tiempo nada más comenzar la inyección de fluidos o la causa de esa sismicidad.

A diferencia de la actividad debida a terremotos de origen natural y tectónico, la sismicidad inducida no puede ser tratada como una actividad estacionaria en el tiempo, tal y como se asume en un estudio probabilista de peligrosidad clásico (PSHA), donde se considera que la tasa de ocurrencia de terremotos es constante, siguiendo un modelo temporal poissoniano homogéneo para cada zona sísmica. En la metodología PISHA (Ec. 1) se considera que los valores de $b$ y la tasa de sismicidad son variables con el tiempo, cobrando además especial importancia los terremotos de bajas magnitudes. Estos cambios de planteamiento entre el enfoque PSHA y PISHA (Ec. 2) se reflejan de este modo en la integral de peligrosidad:

$E_{i}\left(A>A_{0}\right)=\alpha_{i} \int_{M} \int_{R} \int_{\varepsilon} I\left[A>A_{0} \mid m, r, \varepsilon\right] \mathrm{f}(\mathrm{m}) \mathrm{f}(\mathrm{r}) \mathrm{f}(\varepsilon) d m d r d \varepsilon$

$E_{i}\left(A>A_{0}\right)=\int_{T} \int_{M} \int_{R} \int_{\varepsilon} \alpha_{i}(t) I\left[A>A_{0} \mid m, r, \varepsilon\right] \mathrm{f}\left(\mathrm{m}, b_{i}(\mathrm{t})\right) \mathrm{f}(\mathrm{r}) \mathrm{f}(\varepsilon) d t d m d r d \varepsilon$

Dónde:

(2)

- A: representa el parámetro de movimiento con el que expresamos la peligrosidad (habitualmente aceleración pico PGA, o aceleración espectral SA (T).

- A0: es el valor fijado de movimiento cuya excedencia se evalúa.

- Alfa: es la tasa anual de ocurrencia de sismos y se considera constante dentro de una misma fuente.

- $f(m)$ : se refiere a la función de densidad de probabilidad de la magnitud dentro de cada fuente. Describe la probabilidad de que ocurra un terremoto de una determinada magnitud en la fuente sísmica y representa directamente el denominado efecto de la fuente.

- $f(r)$ : se refiere a la función de densidad de probabilidad de la distancia. Describe la probabilidad de que si se produce un terremoto en la fuente, su foco se encuentre a una distancia $r$ del emplazamiento de cálculo. Esta función representa 
la posible localización de un sismo dentro de la fuente sísmica y se relaciona con el efecto de la atenuación del movimiento a lo largo del trayecto entre la fuente y el emplazamiento.

- $f(E p s i l o n)$ : está relacionada con la función de densidad de probabilidad asociada a la incertidumbre del movimiento que puede registrarse en el emplazamiento debido a un sismo determinado. Así, dadas una magnitud $\mathrm{m}$ y una distancia $r$, cabe esperar distintos niveles de movimiento dada la aleatoriedad del mismo, que se considera mediante una distribución log normal de y en base a observaciones empíricas. El parámetro espsilon indica precisamente el número de desviaciones estándar que se consideran para estimar $\mathrm{y}$.

- $\quad I[A>A 0 \mid m, r, e p s i l o n]:$ es un término de probabilidad que refleja si se produce o no excedencia del nivel de movimiento prefijado al calcular el movimiento esperado para unas variables, y determinadas. Es por tanto un término que toma dos valores: 1 cuando sí se produce excedencia y 0 en caso contrario.

- La integral triple se extiende al rango de variación de las tres variables ( $m, r$ y epsilon) y se resuelve numéricamente, dado que en la mayor parte de los casos no existe solución analítica. Sumando la contribución de todas las zonas se obtiene la tasa anual de excedencia del movimiento o peligrosidad sísmica expresada en términos anuales.

- El parámetro t representa el tiempo y solo aparece en el la Ec. (2) para el caso de PISHA

Hasta ahora, son diferentes los trabajos que se pueden encontrar en la literatura referentes a la inclusión de sismicidad inducida por diferentes causas antrópicas en un estudio probabilista de peligrosidad. Convertito et al. (2012) describen un análisis de peligrosidad sísmica probabilista dependiente del tiempo para sismicidad inducida (ISHA), combinando los datos y características de la sismicidad inducida por la inyección y extracción de fluido con un modelo de movimiento fuerte local. Por otro lado, Bourne et al. (2015) plantea una serie de consideraciones a tener en cuenta en la evaluación de la peligrosidad sísmica incluyendo sismicidad inducida. Recientemente, se ha publicado el mapa de peligrosidad para el centro y oeste de Estados Unidos incluyendo la sismicidad inducida por las actividades petroleras de la zona (Petersen et al. 2017)

Según sea la actividad antrópica que influya en la sismicidad de una zona, habrá que considerar un enfoque diferente para su inclusión en el cálculo, ya que el mecanismo por el cual se inducen los terremotos es diferente para cada tipo de actividad antrópica. Para la estimación de la peligrosidad sísmica se deben incluir, además de las características tectónicas, los parámetros propios de la actividad antrópica (operaciones de inyección, llenado, etc)

Entre los principales aspectos identificados por diferentes autores a tener en cuenta para la inclusión de la sismicidad inducida en un estudio de peligrosidad sísmica cabe destacar:

- Magnitud mínima: Tradicionalmente en un estudio PSHA clásico se suele fijar como magnitud mínima
$M=5$, ya que sismos por debajo de esta magnitud no suelen generan daños. Sin embargo los eventos inducidos pueden ser de menores magnitudes y se producen, en general, a profundidades más superficiales, por lo que pueden causar movimientos fuertes del terreno a distancias epicentrales más cortas. Hasta ahora no se tiene un conocimiento preciso de la magnitud mínima capaz de causar daños para este tipo de eventos y por tanto que puedan contribuir a la peligrosidad sísmica.

- Magnitud máxima esperada: Es una de las cuestiones más discutidas y a la que, por el momento, no se ha llegado a ningún tipo de consenso.

- Valor del parámetro b: Son diferentes los estudios en los que se ha visto que el valor de b en casos de sismicidad inducida tiende a tener valores mayores, próximos a 2, frente a los casos de sismicidad natural o tectónica, en los que la pendiente de la recta de GR representada por el parámetro b suele ajustarse a valores del orden de 1.0.

- Modelo de movimiento fuerte: Se deben utilizar modelos que cubran rangos de magnitudes bajas así como distancias hipocentrales cercanas para considerar la sismicidad superficial inducida por las operaciones humanas.

- Efecto sitio.

\section{Caso de estudio: C.P. Pirrís}

El C.P. Pirrís, localizado en Costa Rica, en la zona del Pacífico Central, es uno de los 11 centros de producción hidroeléctricos pertenecientes al Instituto Costarricense de Electricidad (ICE). Se encuentra en una zona montañosa en donde diferentes estudios geológicos han demostrado la existencia de fallamiento activo en los alrededores del embalse (Informe 2006, Red sismológica para el P.H. Pirris). Por ello, es importante monitorear y controlar la ocurrencia de sismicidad en torno al embalse, en especial aquella que pueda ser provocada por el llenado del mismo.

Se trata de una presa de gravedad construida con concreto compactado con rodillo (RCC), de $113 \mathrm{~m}$ de altura, $266 \mathrm{~m}$ de longitud y con la cresta a la elevación de $1208 \mathrm{msnm}$, que crea un embalse de regulación mensual. Este centro de generación hidroeléctrica tiene una capacidad de generación de 130 MW. Consta, además de la presa, de un túnel de conducción de 11 $\mathrm{km}$ de longitud y una casa de máquinas. El embalse tiene un volumen de $36 \times 10^{6} \mathrm{~m}^{3}$, se extiende por 3,5 $\mathrm{km}$ en sentido $\mathrm{E}-\mathrm{W}$ y $1,2 \mathrm{~km}$ en sentido $\mathrm{N}-\mathrm{S}$, con una profundidad máxima de unos $80 \mathrm{~m}$ (Barquero and Alvarado 2012). En la Tabla 1 se resumen las principales características del embalse.

El embalse, cuyo llenado se completó en el año 2011, es de tipo estacional. Ésto significa que, durante un periodo de 4 a 6 meses, coincidentes con la estación lluviosa, se acumula agua para ser utilizada en el posterior periodo estacional seco. (Informe área de Ingeniería Hidráulica, ICE agosto 2012).

Con el fin de controlar el comportamiento de las diferentes estructuras del C.P. Pirrís, se han ido colocando desde la fase constructiva de la presa (año 2009) distintos instrumentos de medición para realizar 
tanto controles de tipo estructural como geotécnico, los cuales continúan realizándose ahora en fase de operación.

Tabla 1: Resumen de las principales características del embalse de Pirrís

\begin{tabular}{c|c} 
Año de inicio & 2011 \\
\hline Área del embalse & $114 \mathrm{Ha}$ \\
Volumen total & $36 \mathrm{hm} 3$ \\
Cota máxima & $1205 \mathrm{msnm}$ \\
Cota mínima & $1160 \mathrm{msnm}$ \\
Caudal promedio anual & $9.8 \mathrm{~m} 2 / \mathrm{s}$ \\
Tipo de regulación & Estacional \\
Prof. Máxima & $80 \mathrm{~m}$
\end{tabular}

\subsection{Contexto tectónico}

La Planta de Pirris se encuentra en una zona tectónicamente compleja. La sismicidad en sus alrededores está asociada principalmente a dos tipos de fuentes: por un lado, la sismicidad generada por la zona de subducción y por otro, la sismicidad generada por fallamiento local (Fig. 1).

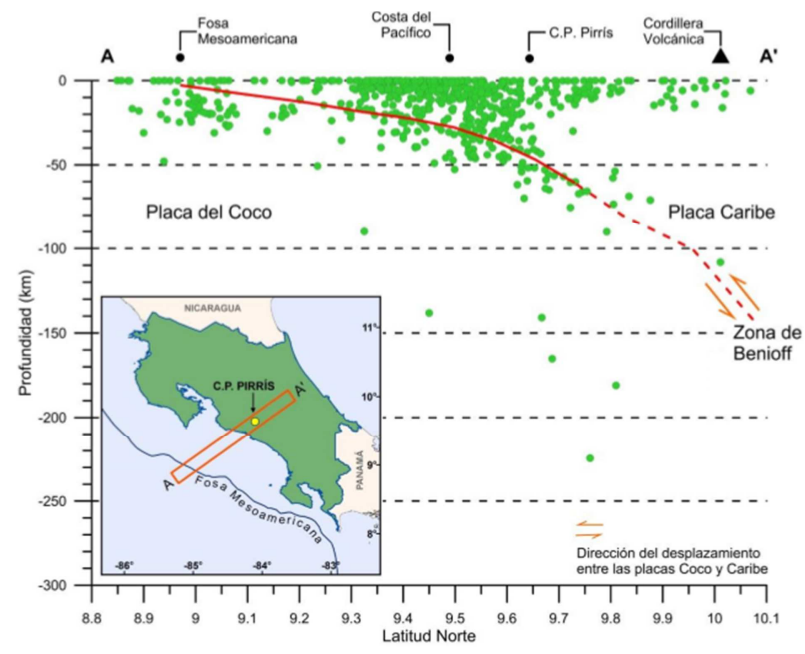

Figura 1: Perfil de la zona de subducción (Imagen tomada de Informe PH Pirris, 2012).

El primer tipo de sismicidad está asociada al proceso de subducción de la placa del Coco bajo la placa Caribe, que se extiende en profundidad desde la fosa hacia el interior del país, hasta la conocida "zona de Benioff."

Los esfuerzos compresivos generados por el proceso de subducción entre ambas placas, originan en la zona del Pacífico Central del país, un complejo sistema de fallas de carácter regional, predominantemente de tipo inverso, a nivel de la corteza continental (Tabla 2). De ellas la más importante es la llamada "Falla Longitudinal de Costa Rica", localizada principalmente en la región del Pacífico Central y Sur de Costa Rica.

El segundo tipo de sismicidad existente en los alrededores del embalse, se asocia con la tectónica y fallas locales. Este tipo de sismos se producen por los esfuerzos internos propios de la placa, o pueden ser sismos disparados o inducidos, producidos por actividad antropogénica, como la asociada a embalses y presas. Existen diferentes trabajos y estudios sobre el análisis sismo-tectónico de las fuentes sísmicas asociadas con fallamiento local de la zona del Pacífico Central. La información tectónica proviene principalmente de los estudios geológico-tectónicos de Mora (1984), Barquero et al. (2001), Denyer et al. (2003) y Barquero and Climent (2006), aunque por el momento no existe ningún estudio específico reciente para las fallas más cercanas al embalse de Pirrís. Por tanto no se tiene la información suficiente como para modelizarlas como fuentes independientes, pues no conocemos su geometría, valores de slip rates, etc.

Tabla 2: Principales fallas regionales del Pacifico Central

\begin{tabular}{c|c|c} 
Falla & Tipo & Longitud mínima $(\mathrm{km})$ \\
\hline Longitudinal & Inversa & Mayor a 200 \\
Damas & Inversa & 25 \\
Tierras Morenas & Inversa & 10 \\
La Faralla & Inversa & 15 \\
Paquita & Inversa & 20 \\
Naranjo & Inversa & 12 \\
Pirrís-Pagolín & Normal & 16 \\
Quepos & Normal-dextral & 10
\end{tabular}

Algunas de las fallas locales que se encuentran dentro de la zona de estudio atraviesan el embalse de Pirrís, cortándolo en sentido NW-SE y NE-SW, principalmente (Fig. 2). Todas ellas son de longitudes pequeñas, no alcanzándose en casi ningún caso longitudes mayores a $5 \mathrm{~km}$ (Tabla 3). Con estas longitudes, se ha realizado una estimación utilizando la relación de Wells and Coppersmith (1994) para conocer cuál es la magnitud máxima esperada en cada falla suponiendo que rompiera en toda su longitud. La falla con el potencial para generar el mayor sismo en la zona es la falla de Zapote con una Mmax $5.8(\mathrm{Mw})$, pues es la falla de mayor longitud. Le sigue la falla de San Rafael, con una magnitud máxima Mw 5.7. Cabe también destacar la falla La Isla con una Mmax estimada de 5.4, la cual cruza longitudinalmente el embalse.

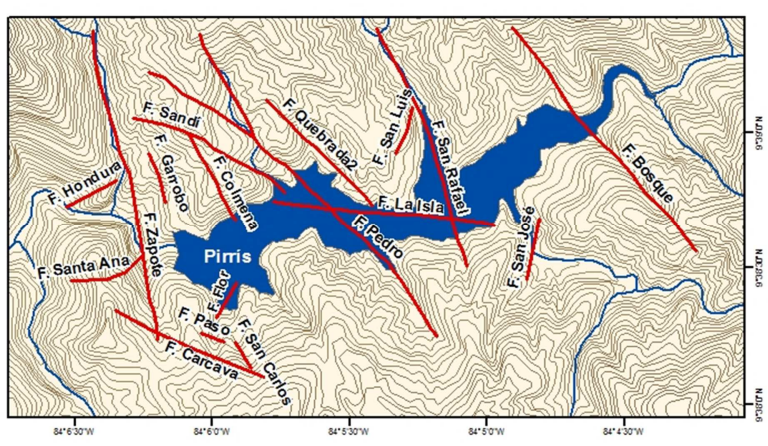

Figura 2: Distribución espacial de las trazas de las fallas locales en el embalse de Pirrís.

Históricamente, no se tienen registros de sismos que hayan sido originados por fallas locales cercanas (menos de $25 \mathrm{~km}$ ) al P.H. Pirrís; solo se han presentado terremotos cuya fuente ha sido el proceso de 
subducción de la placa del Coco bajo la placa Caribe, siendo el más importante el de 1952 de magnitud 7,0, que alcanzó una intensidad máxima de VII en la parte central interior de Costa Rica (Montero and Climent 1990).

Tabla 3: Principales fallas locales

\begin{tabular}{c|c|c|c|c} 
Nombre & Tipo & Rumbo & $L(\mathrm{~km})$ & Mmax \\
\hline Zapote & Dextral & 350 & 8 & 5.8 \\
Garrobo & Dextral/normal & 340 & 0.8 & 4.7 \\
Colmena & Dextral & 340 & 2.4 & 5.2 \\
Sandí & Dextral & 140 & 2.6 & 5.3 \\
Pedro & Dextral/normal & 310 & 0.6 & 4.6 \\
Quebrada2 & Dextral & 320 & 2 & 5.2 \\
San Luis & Normal & 30 & 0.8 & 4.7 \\
Bosque & Rumbo & 330 & 3.6 & 5.4 \\
San José & Normal & 10 & 0.9 & 4.8 \\
Cárcava & Dextral & 300 & 1.4 & 5.0 \\
San Carlos & Normal & 330 & 1.8 & 5.1 \\
Paso & Inversa & 110 & 0.4 & 4.4 \\
Flor & Sinestral & 15 & 0.6 & 4.6 \\
La Isla & Normal & 100 & 3 & 5.4 \\
San Rafael & Sinestral & 150 & 6 & 5.7
\end{tabular}

\subsection{Actividad y operaciones en el embalse}

Tras finalizar la construcción de la represa, el 9 de marzo de 2011 se iniciaron las operaciones de llenado del embalse, alcanzándose el nivel máximo el día 16 de Agosto de ese mismo año. Tras su llenado, los niveles de agua en el embalse han ido variando, siguiendo una tendencia más o menos cíclica (Fig. 3) con diferentes disminuciones del nivel medio del agua en diferentes épocas de los sucesivos años, coincidiendo normalmente con los meses de julio y agosto que es cuando se presenta una disminución relativa de la precipitación y suele coincidir con el llamado "Veranillo". Concretamente, en julio de 2015, se produjo un desembalse con el objetivo de revisar el túnel de conducción. Muchas de estas fluctuaciones en el nivel de agua pueden tener repercusión en la sismicidad de la zona, hecho que se intentará analizar y cuantificar en futuros análisis.

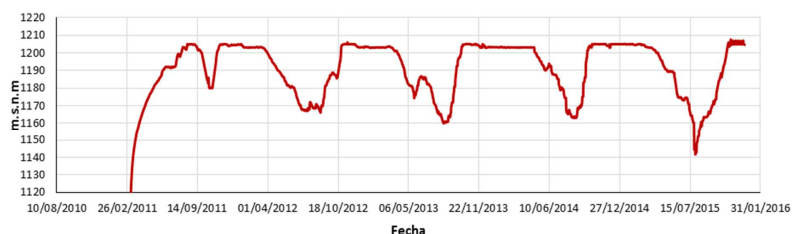

Figura 3: Variación diario del nivel de agua en el embalse.

\subsection{Datos sísmicos. Red sismológica local}

La red sismológica local de Pirrís fue instalada en febrero de 2008, antes de que se empezara con el llenado del embalse, para poder monitorear así la actividad sísmica un tiempo antes del llenado del mismo, y poder comparar esa actividad con la que podría ser disparada tras el llenado.

Originalmente, la red constaba de 6 estaciones, ampliándose en 2013 con la instalación de tres nuevas estaciones, consiguiendo así una mejor cobertura de la zona de influencia (Fig. 4).

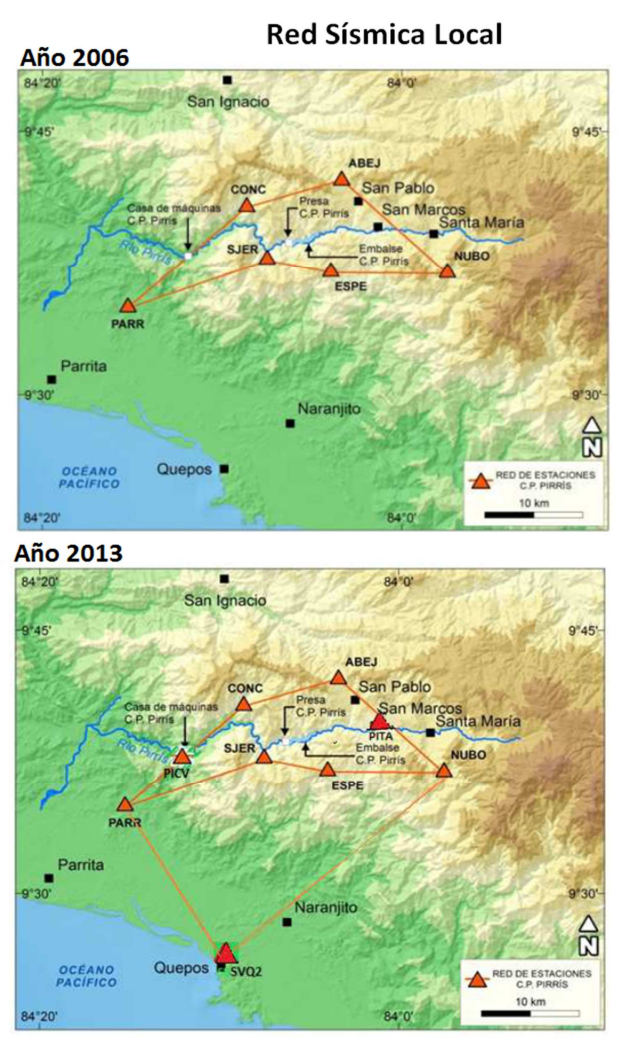

Figura 4: Evolución de la red sísmica local: red original (año 2006) y su densificación en 2013 con la instalación de tres nuevas estaciones (año 2013).

Además de la red sismológica local, desde septiembre de 2011, se cuenta con un sistema de monitoreo acelerográfico en el sitio de la presa para controlar el comportamiento dinámico de la estructura y garantizar su seguridad actual ante la ocurrencia de posibles sismos. La red acelerométrica consta de 7 acelerógrafos ubicados en diferentes niveles del cuerpo de la presa (Fig. 5) que registran en las tres componentes del espacio (Climent and Piedra 2012).

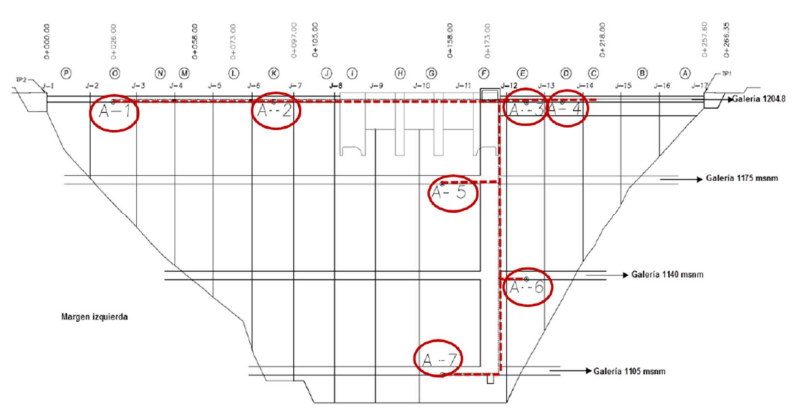

Figura 5: Ubicación de los sensores acelerométricos en la presa del C.P. Pirrís. 


\section{Análisis de la sismicidad}

Se ha realizado un primer análisis de la sismicidad regstrada por la red sísmica local en el entorno del embalse del C.P. de Pirrís diferenciándose entre los pediodos de tiempo antes, durante y después de la fase de llenado, con el objetivo de diferenciar algún cambio en el comportamiento o patrón de sismicidad de la zona (Fig. 6). Con el fin de completar este estudio de sismicidad alrededor del embalse durante el periodo antes del llenado, y conocer cuál era la sismicidad de fondo en la zona, se cuenta con los datos registrados por la Red Sismológica Nacional (RSN) desde el año 1977 hasta el momento de la instalación de la red sismológica local. La mayor parte de los análisis realizados se han hecho con el paquete de herramientas de Mapseis.

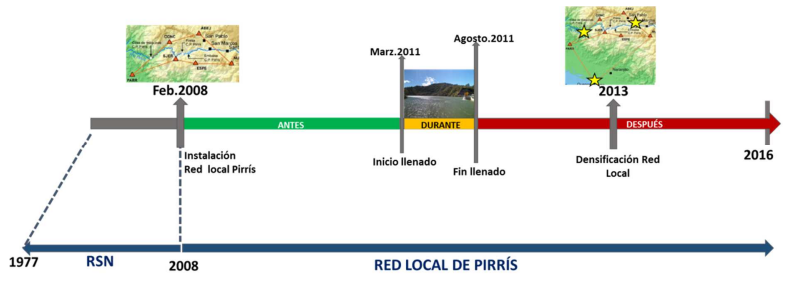

Figura 6: Línea del tiempo donde se muestra el momento de instalación de la red sismológica local, la densificación de la

misma red así como el periodo del llenado del embalse.

\subsection{Análisis descriptivo}

Para efectos del análisis propuesto, se han utilizado únicamente los eventos registrados por la red local de Pirrís descrita anteriormente, desde su instalación en Febrero de 2008, hasta finales del 2015. Este rango de tiempo cubre el periodo antes del llenado del embalse, así como el periodo durante y después del llenado. Los registros han sido homogeneizados a magnitud momento $\left(\mathrm{M}_{\mathrm{w}}\right)$ a través de la relación propuesta por Rojas et al. 1993 (Ecs. 3 y 4).

$M_{S}=-4.165+1.783 M_{L}$

$M_{W}=2.251+0.655 M_{S}$

Dónde:

- $\quad \mathrm{M}_{\mathrm{L}}=\mathrm{M}_{\mathrm{C}}=\mathrm{M}_{\mathrm{d}}$.

- $\quad \mathrm{M}_{\mathrm{S}}$ : magnitud de las ondas superficiales.

- $\quad \mathrm{M}_{\mathrm{L}}$ : Magitud local o de Richter.

- $\quad \mathrm{M}_{\mathrm{C}}$ : magnitud de coda.

- $\quad \mathrm{M}_{\mathrm{d}}$ : magnitud duración.

Además de la diferenciación temporal en función de la fase de llenado, se han considerado diferentes áreas de estudio dependiendo del análisis realizado, que van desde los $5 \mathrm{~km}$ hasta los $20 \mathrm{~km}$ alrededor del centroide del embalse. Únicamente se han considerado los eventos superficiales (profundidades $\leq 20 \mathrm{~km}$ ) y con un RMS en su localización menor de 0.4. Las características de cada uno de los catálogos utilizados en función del área de estudio escogida se recogen en la Tabla 4.

En la Figura 7 se muestran los histogramas que representan el número total de eventos en función de la magnitud, profundidad y horas del día, respectivamente, para el área de $5 \mathrm{~km}$ alrededor del embalse. De esta forma se puede ver rápidamente cuales son las características predominantes del catálogo utilizado.

Tabla 4: Resumen de los datos del catálogo utilizado en función del área de influencia considerada

\begin{tabular}{c|c|c|c}
$\begin{array}{c}\text { CATÁLOGO } \\
R E D \text { PIRRÍS }\end{array}$ & $\begin{array}{c}A ́ R E A \text { de 20 } \\
\mathrm{km}\end{array}$ & $\begin{array}{c}\text { ÁREA de 10 } \\
\mathrm{km}\end{array}$ & $\begin{array}{c}\text { ÁREA de } 5 \\
\mathrm{~km}\end{array}$ \\
\hline $\begin{array}{c}\text { Rango de } \\
\text { Magnitudes }\end{array}$ & $0.0-5.2$ & $0.0-4.8$ & $0.0-4.2$ \\
$\begin{array}{c}\text { Rango de } \\
\text { profundidades }\end{array}$ & & $\leq 20 \mathrm{~km}$ & \\
RMS & & $\leq 0.4$ & \\
$\mathrm{n}^{\circ}$ total & & 1354 & 561 \\
registros & 2403 & & \\
periodo de & $09 / 02 / 2008$ & $09 / 02 / 2008$ & $09 / 02 / 2008$ \\
tiempo & $19 / 12 / 2015$ & $16 / 12 / 2015$ & $16 / 12 / 2015$
\end{tabular}
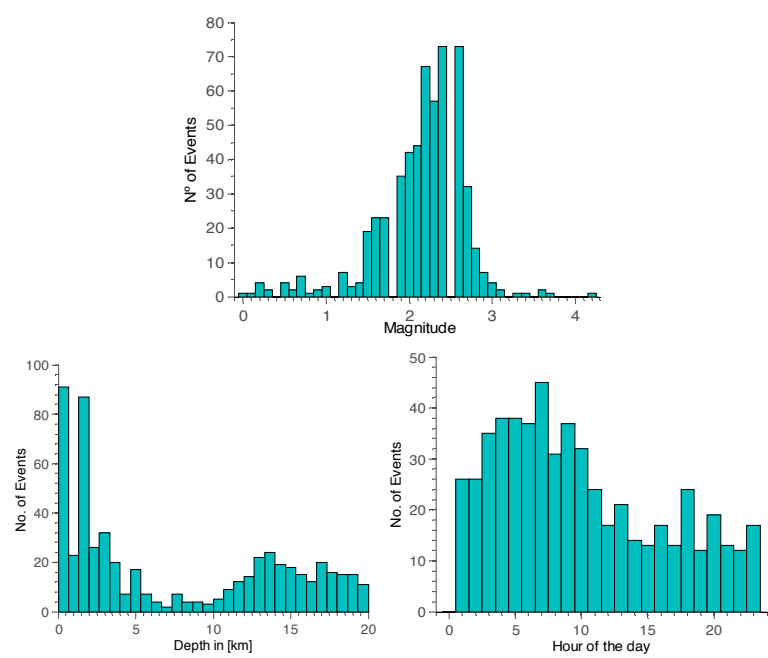

Figura 7: Histogramas de magnitud, profundidad y registro horario de eventos.

El mayor evento de la serie $\left(M_{w} 4.2\right)$ dentro del área epicentral de $5 \mathrm{~km}$, se registró en diciembre de 2011, casi 5 meses después del final del llenado del embalse a una distancia aproximada de $2 \mathrm{~km}$ del sitio de presa y profundidad de $2 \mathrm{~km}$.

Analizando el número total de eventos, se comprueba que para las diferentes áreas de influencia escogidas, el número de eventos tras el llenado es muy superior a los registrados antes y durante el llenado. Sin embargo, dado que la ventana de tiempo que comprende cada periodo analizado es muy diferente, el número neto de eventos no es una variable del todo comparable (barras en Figura 8). Lo que sí se puede comparar, por estar normalizada, es la tasa de sismos diaria (línea negra) que muestra un claro ascenso progresivo tras el comienzo de las operaciones de llenado (Fig. 8).

Analizando el porcentaje de eventos registrados en función de diferentes rangos de magnitud, se comprueba que antes del llenado el mayor porcentaje de eventos registrados son de magnitudes $\leq 1.9$. Sin embargo, tras el llenado, los eventos que mayoritariamente se registran son de magnitudes $\geq 2.0$ (Fig. 9) 

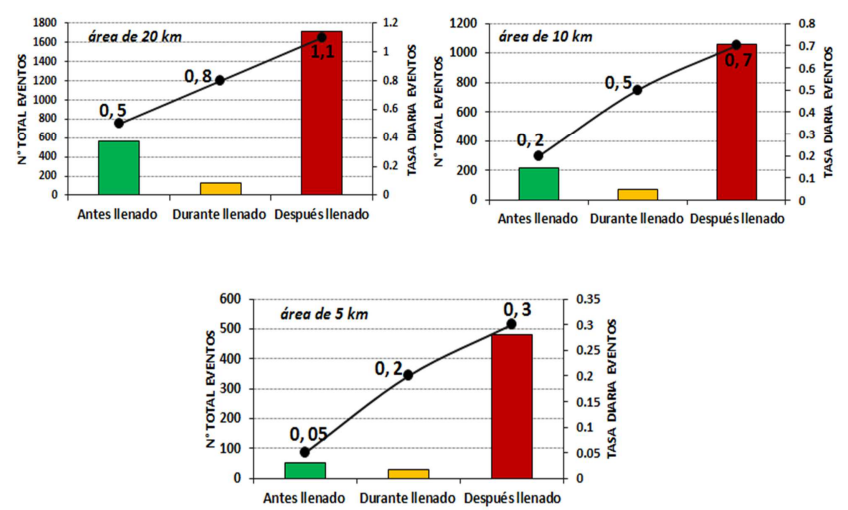

Figura 8: Número total de eventos (barras) y tasa diaria de sismos (línea negra) diferenciando entre antes, durante y después del llenado del embalse.

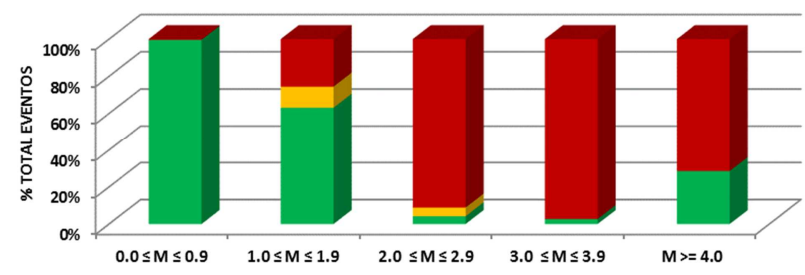

Figura 9: Porcentaje de eventos registrados para diferentes rangos de magnitud diferenciando el periodo de antes del llenado (verde), durante (amarillo) y después (rojo) del llenado.

Tras analizar más de 100 casos, Guha and Patil (1990) clasifican la sismicidad inducida en tres categorías en función de las magnitudes registradas: sismicidad intensa (magnitud superior a 6.0), moderada-débil (magnitud entre 3.1-5.9) y micro-temblores (magnitud inferior a 3.0). Siguiendo esta clasificación, el caso de la sismicidad asociada a Pirrís se encontraría dentro de la tercera categoría (micro-temblores) durante el llenado y de la segunda categoría (sismicidad moderada-débil) tras el llenado.

\subsection{Análisis espacio-temporal}

$\mathrm{Si}$ se analiza la distribución de sismicidad temporalmente, diferenciándose los tres periodos anteriores, antes, durante y después del llenado, se puede ver que, tras el inicio del llenado, la actividad sísmica en la zona empezó a aumentar, registrándose un nivel de sismicidad mayor alrededor del mismo. En la Figura 10, se muestra el número de eventos al mes junto con el número acumulado de sismos dentro de una distancia epicentral de 5,10 y $20 \mathrm{~km}$, respectivamente, diferenciándose los periodos antes, durante y después del llenado (colores verde, naranja y rojo respectivamente). En los tres gráficos se ve claramente el acusado aumento de la sismicidad tras el llenado del embalse, así como en el periodo de agosto de 2014, coincidiendo con un importante descenso del nivel de agua en el embalse.

En la zona más cercana al embalse ( $5 \mathrm{~km})$, se detecta un aumento de pequeños eventos (Mw 1.5-3.0) espacialmente agrupados y muy superficiales (profundidades menores de $5 \mathrm{~km}$ ). Esta sismicidad coincide justamente con las primeras operaciones realizadas en el embalse, en las que puede apreciar un claro aumento de la pendiente del número acumulado de sismos. La magnitud máxima registrada en este primer cluster es de Mw 4.2 y corresponde con el máximo evento de la serie en el área de $5 \mathrm{~km}$ alrededor del embalse (Fig. 10).

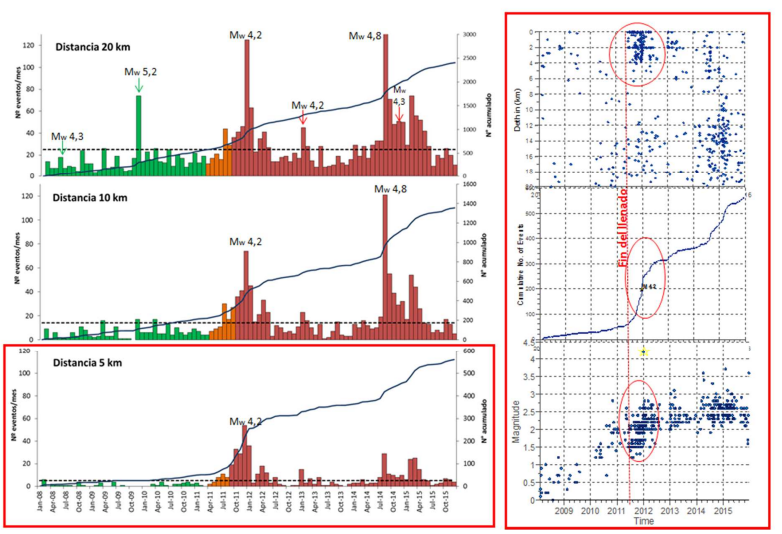

Figura 10: Evolución temporal de la serie sísmica considerando diferentes áreas $(20,10$ y $5 \mathrm{~km}$ alrededor del embalse). Las barras representan el número de eventos al mes y la línea azul el número acumulado de sismos. Para el área de $5 \mathrm{~km}$ se muestra la distribución de epicentros en el tiempo en función de la profundidad, número acumulado y magnitud (figuras de la parte derecha).

Si se analiza la distribución espacial de los eventos más someros $(\mathrm{h} \leq 5 \mathrm{~km})$ en cada una de las fases estudiadas (Fig. 11), cabe destacar cómo, antes del llenado, los epicentros se encuentran más dispersos y conforme empiezan las operaciones de llenado, se puede apreciar un ligero aumento de la concentración de eventos en la zona más próxima al embalse, que se intensifica tras el llenado. Después del llenado, la concentración de sismos es mayor, aumentando también la magnitud, que llega a ser de Mw 4.2 para el mayor evento de la serie.

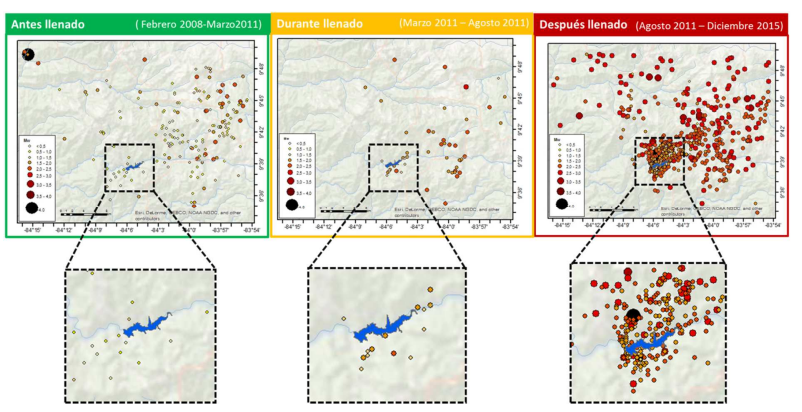

Figura 11: Distribución espacial de eventos antes, durante y después del llenado del embalse.

Con el objetivo de conocer la evolución espaciotemporal de la sismicidad, se ha elaborado el gráfico $\mathrm{r}$ - $\mathrm{t}$ (distancia epicentral-días) (Fig. 12). Como origen de distancias se ha elegido el centroide del embalse y el origen de tiempo se ha fijado en el momento en que se inició el llenado. Se puede ver que, justo al finalizar el llenado, los eventos se concentran a cortas distancias y conforme pasa el tiempo, los eventos empiezan a registrarse a distancias epicentrales ligeramente mayores (entre 6 y $12 \mathrm{~km}$ ). Éste hecho, podría ser un indicativo de un cambio de fracturación en el medio, debido a una alteración de los esfuerzos en la zona, lo 
cual podría venir explicado por una posible dispersión de la presión de poros por el medio.

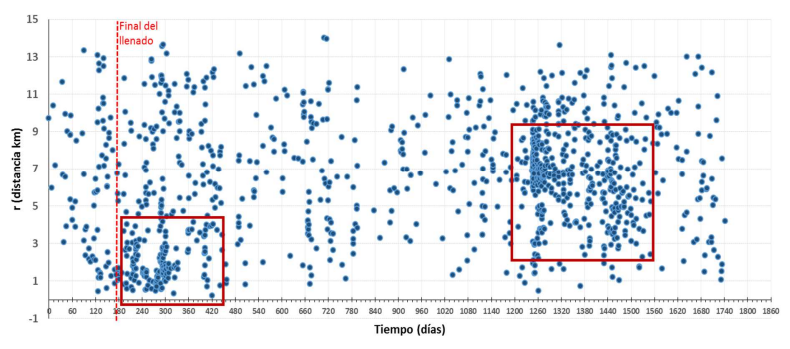

Figura 12: Gráfico r-t.

\section{Análisis de parámetros sísmicos}

\subsection{Distribución frecuencia-magnitud. Ley de GR}

Con el objetivo de caracterizar la sismicidad en la zona, se aplica la ley de Gutenberg-Richter (G-R) a la serie de datos y se analizan los parámetros resultantes del ajuste. Además se hace un análisis de la tendencia de la variación anual de los parámetros propios de la ley a través de una regresión lineal.

La relación de $\mathrm{G}-\mathrm{R}$ (Ion $\mathrm{N}=\mathrm{a}-\mathrm{bm}$ ) representa la relación entre el número de sismos y su magnitud (Gutenberg and Richter 1944). N representa el número de terremotos de magnitud igual o mayor que $\mathrm{m}, \mathrm{y}$ a es el número de terremotos de magnitud $\mathrm{M}=0$. $L a$ variable $b$ describe la relación entre terremotos pequeños y grandes y expresa la pendiente del mejor ajuste entre el número de terremotos a una magnitud dada y la magnitud. En el caso de la sismicidad natural, el valor de $b$ suele estar comprendido entre 0.8-1.0 y varía entre los diferentes tipos de regiones. Sin embargo, se ha observado que, para diferentes situaciones de sismicidad inducida, el parámetro $b$ suele tener un valor mayor y más cercano a 2.0 (Gupta et al. $1972 \mathrm{a}, \mathrm{b}$ ).

En este caso, se ha llevado a cabo un análisis de los parámetros de la recta de G-R con los datos de la Red Sismológica de Pirrís, tomando el área de 20, 10 y 5 km alrededor del embalse. Para ello se ha utilizado el programa Mapseis que realiza el ajuste por máxima verosimilitud y se han aplicado dos métodos diferentes para la estimación de Mc: el método EMR y el de máxima curvatura (Fig. 13).

\begin{tabular}{|l|l|l|l|l|l|l|l|l|}
\hline \multirow{2}{*}{} & \multicolumn{4}{|l|}{ MÉTODO 1 (Máx. curvatura) } & \multicolumn{4}{l|}{ MÉTODO 2 (EMR) } \\
\cline { 2 - 9 } & $\boldsymbol{b}$ & $\boldsymbol{a}$ & $\boldsymbol{a}$ (anual) & Mc & $\boldsymbol{b}$ & $\boldsymbol{a}$ & $\boldsymbol{a}$ (anual) & \multicolumn{1}{l|}{$\boldsymbol{M}$} \\
\hline $\mathbf{2 0} \mathrm{km}$ & $1.72+-0.36$ & 7.28 & 6.39 & $2.5+-0.05$ & $2.21+-0.15$ & 8.66 & 7.76 & $2.6+-0.07$ \\
\hline $\mathbf{1 0} \mathrm{km}$ & $1.72+-0.27$ & 6.96 & 6.06 & $2.4+-0.06$ & $2.5+-0.17$ & 9.13 & 8.23 & $2.6+-0.06$ \\
\hline $\mathbf{5 ~ k m}$ & $2.05+-0.56$ & 7.38 & 6.49 & $2.5+-0.14$ & $2.53+-0.3$ & 8.70 & 7.81 & $2.6+0.01$ \\
\hline
\end{tabular}

Figura 13: Parámetros de GR para diferentes zonas de influencia con dos metodologías diferentes.

Por otro lado, tomando exclusivamente el área de 20 $\mathrm{km}$, se han diferenciado las tres etapas de antes, durante y después del llenado obteniéndose en cada una los parámetros de la recta de GR resumidos en la Figura 14. En este caso se ve claramente el cambio en el valor de $b$ antes y tras el inicio del llenado, pasando de un valor más próximo a la unidad (caso de sismicidad natural) a un valor mayor y próximo a 2 (tal y como se han observado en otros casos de sismicidad inducida).
Asimismo se refleja un importante ascenso de la tasa anual de sismos (a (anual)) variando de aproximadamente 3 eventos al año antes del llenado a 8 tras el inicio del llenado. Aplicando ambos métodos se comprueba que los resultados convergen dando una mayor robustez a las estimaciones obtenidas.

\begin{tabular}{|l|c|c|c|c|c|c|c|c|}
\hline \multirow{2}{*}{ AREA 20 km } & \multicolumn{4}{|c|}{ MÉTODO 1 (Máx. curvatura) } & \multicolumn{4}{c|}{ MÉTOOD 2 (EMR) } \\
\cline { 2 - 9 } & $b$ & $a$ & $a$ (anual) & Mc & $b$ & $a$ & $a$ (anual) & Mc \\
\hline Antes & $0.66 \pm 0.06$ & 3.17 & 2.68 & $0.81 \pm 0.15$ & $0.73 \pm 0.11$ & 3.29 & 2.81 & $1.0 \pm 0.25$ \\
\hline Durante & $2.56 \pm 0.96$ & 7.44 & 7.79 & $2.2 \pm 0.18$ & $2.86 \pm 0.93$ & 8.14 & 8.5 & $2.3 \pm 0.13$ \\
\hline Después & $1.99 \pm 0.39$ & 8.04 & 7.4 & $2.5 \pm 0.1$ & $2.27 \pm 0.1$ & 8.82 & 8.18 & $2.6 \pm 0.06$ \\
\hline
\end{tabular}

Figura 14: Parámetros de GR para los periodos temporales de antes, durante y después del llenado.

Además se ha realizado el análisis de la ley de GR de la serie antes del llenado, utilizando únicamente los datos registrados por la RSN para ver la tasa de fondo de la sismicidad natural en la zona y por otro lado, a partir del inicio del llenado, considerando los datos de la Red local de Pirrís. Se comprueba que el valor de b regional antes del llenado es menor que le valor de $b$ tras el inicio del llenado del embalse (Fig. 15).

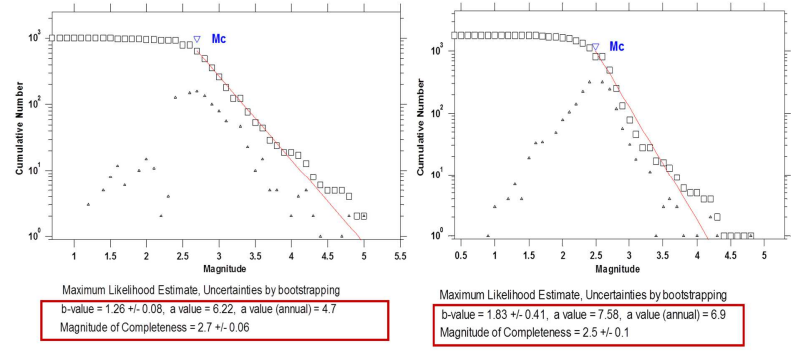

Figura 15: Rectas de GR teniendo en cuenta el catálogo de la RSN hasta el comienzo del llenado (gráfico izquierdo) y utilizando los datos registrados por la Red Sismológica Local de Pirrís tras el inicio del llenado (gráfico derecho).

Por otro lado, se ha intentado analizar la tendencia anual de los parámetros $b$, magnitud máxima teórica y magnitud mínima (Mc), a través de regresión lineal por mínimos cuadrados para estimar el valor de la pendiente (Fig. 16). La tendencia general de los parámetros estudiados es creciente en el tiempo. En cuanto al parámetro $b$, la tendencia ascendente del valor estimado es progresiva en el tiempo, tomando valores más próximos a 2 a partir de 2011 cuando se produce el llenado. Sin embargo, en 2014 el valor estimado es menor, debido a que el ajuste de la recta de GR tiende a ser bilineal, por lo que el valor mostrado en la gráfica no es un valor propio de un buen ajuste de los registros de ese año.

\subsection{Variación temporal del parámetro $b$}

El análisis de la evolución temporal de parámetros sísmicos se realiza a través del método de ventanas deslizantes, a partir del cual se calculan los parámetros para diferentes ventanas temporales cuyo tamaño se fija con el número de eventos. Las ventanas se van desplazando en el tiempo con un solape fijo. Haciendo diferentes pruebas se llega a encontrar la ventana óptima para el análisis temporal de cada parámetro. En este caso, la ventana mínima utilizada para la estimación de $b$ es de 120 eventos con un solape entre ventanas del $10 \%$, obteniéndose un error en la 
estimación del parámetro b menor a 0.5 (Fig. 16). El valor de $b$ se mantiene en la franja de $1.5-2.5$ hasta aproximadamente mediados de 2014, cuya tendencia cambia, empezando a tomar valores superiores (Fig. 17). Este momento de cambio en la estimación de b coincide precisamente con un hecho observado en el análisis espacio-temporal de la sismicidad (gráfico r-t de Figura 11) en donde los eventos empiezan concentrarse a mayores distancias respecto del embalse. Esto podría ser otro indicador que esté informando de una posible dispersión de la presión de poros por el medio, hecho que debe ser analizado con mayor detalle para su confirmación.
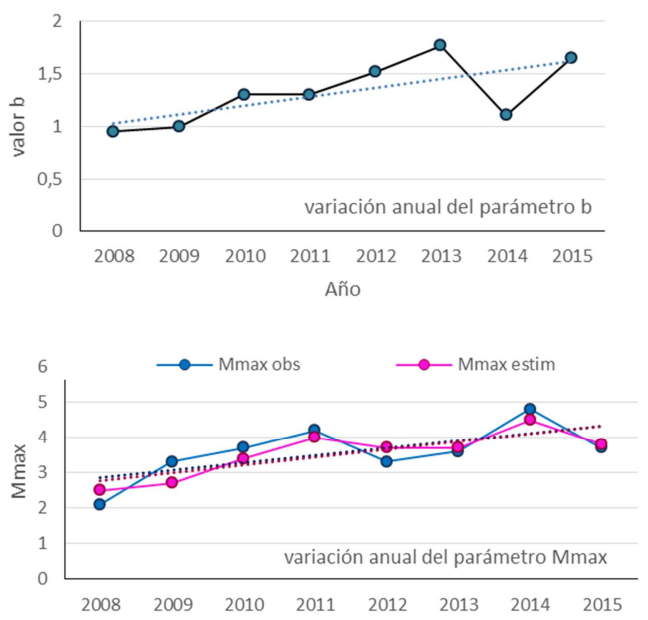

Año

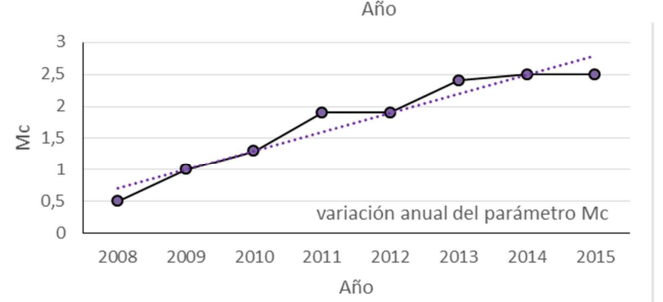

Figura 16: Análisis anual de diferentes parámetros (valor de b, Mmax y Mc).

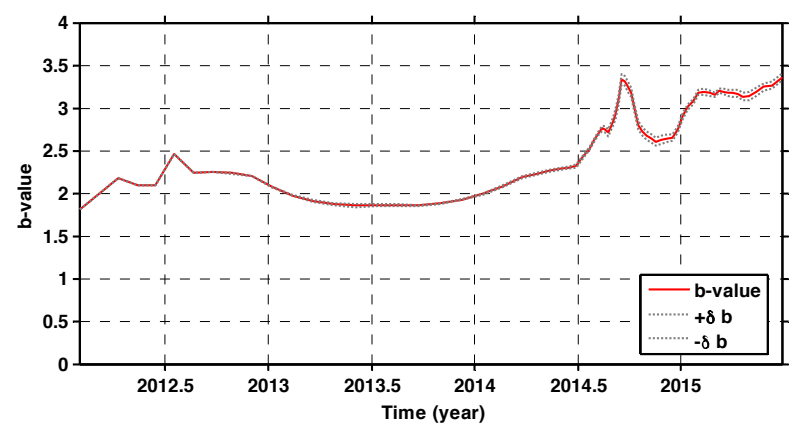

Figura 17: Evolución temporal de b.

\subsection{Relación de la sismicidad con los cambios en el nivel de agua en el embalse}

Diferentes autores (Gupta 1983, Gupta et al. 2002) han puesto de manifiesto que para el análisis de la sismicidad asociada a embalses hay que tener en cuenta factores como la velocidad de variación del nivel del agua, el tiempo de duración del proceso de carga, el nivel máximo alcanzado y el periodo de tiempo durante el cual se mantiene este nivel máximo, pues son factores que están estrechamente relacionados con la posible actividad sísmica en la zona. En nuestro caso de estudio y viendo la gráfica de los cambios de nivel en el embalse, se aprecia que el nivel máximo del mismo se suele mantener entre 5 a 6 meses, coincidiendo normalmente con los meses de julio y agosto. Los cambios más acusados en el nivel de agua se producen en un tiempo de 2 a cuatro meses, y las variaciones nunca superan los $15 \mathrm{~m}$ de diferencia en el nivel del embalse al día y de $22 \mathrm{~m} / \mathrm{mes}$ tras el primer llenado.

Se puede apreciar que hay una clara correlación entre el aumento de la sismicidad y el primer llenado del embalse (Fig. 18). Sin embargo, lo que no se ve tan claro es que exista una correlación entre la sismicidad y los sucesivos cambios en el nivel del embalse. Por ello sería importante cuantificar esta posible correlación

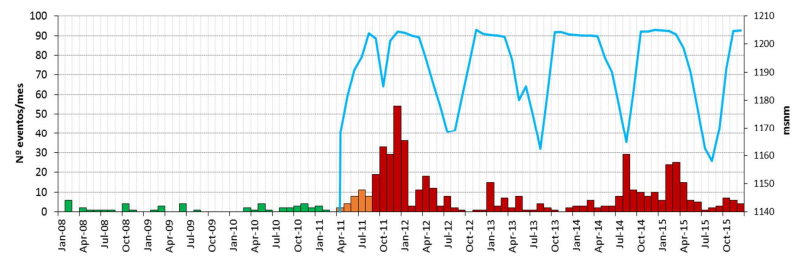

Figura 18: Nivel del embalse al mes (línea azul) junto con la sismicidad registrada al mes (columnas).

\section{Estimación de la magnitude maxima}

La cuestión de la magnitud máxima que puede ser atribuida a casos de sismicidad asociada a grandes embalses es difícil de clarificar. Una opción es fijarse en otros casos y ver que magnitudes máximas se han alcanzado en ellos. En el caso de sismicidad natural, en general una magnitud de 9-9.30 es aceptada como máxima observada. Considerando todos los casos hasta ahora conocidos de sisimicidad disparada por embalses, solo en cuatro ocasiones se han alcanzado magnitudes de 6.0 - 6.3. Se podrían aceptar tales valores de magnitud como el umbral de la Mmax esperada en estos casos (ICOLD)

Con base en los sismos históricos e instrumentales, el sismo máximo probable para el proceso de subducción es un evento de magnitud 7.0. Las intensidades máximas probables para la zona son del orden de I VIII (EMS). Con esta intensidad se esperaría una sacudida del suelo lo suficientemente fuerte como para originar deslizamientos superficiales en zonas inestables y con fuerte pendiente.

Para el caso estudiado se han probado las metodologías propuestas por Kijko (2004; 2011).

Se ha realizado la estimación de la magnitud máxima teniendo en cuenta toda la serie sísmica, así como los periodos de antes, durante y después del llenado, considerados de forma independiente. Los resultados obtenidos con cada uno de los métodos aplicados, así como el valor promedio de todas las estimaciones, son presentados en la Tabla 5 resaltando la magnitud máxima observada en cada caso.

Se puede comprobar que el promedio de las máximas estimadas es mayor a la magnitud máxima estimada en todos los casos, siendo está diferencia mayor para el caso del periodo de antes del llenado. 
Tabla 5: Magnitudes máximas estimadas junto con un promedio de todas las estimaciones y la magnitud máxima observada. Análisis realizado para toda la serie así como para el periodo de antes, durante y después del llenado independientemente.

\begin{tabular}{c|c|c|c|c} 
& \multicolumn{4}{|c}{ Mmax $\pm \sigma$} \\
& Todo & Antes & Durante & Después \\
\hline $\begin{array}{c}\text { Mmax } \\
\text { Observada } \\
\text { N-P-G }\end{array}$ & 5.2 & 5.2 & 2.9 & 4.8 \\
$\begin{array}{c}\text { Order Statistics } \\
\text { CDF }\end{array}$ & $5.4 \pm 0.3$ & $5.6 \pm 0.5$ & $3.0 \pm 0.2$ & $5.0 \pm 0.2$ \\
$\begin{array}{c}\text { Based on 5 } \\
\text { largest mag } \\
\text { Kijko-Sellevoll }\end{array}$ & $5.4 \pm 0.3$ & $5.7 \pm 0.5$ & $3.0 \pm 0.1$ & $5.0 \pm 0.2$ \\
$\begin{array}{c}\text { Tate-Pisarenko- } \\
\text { Bayes }\end{array}$ & $6.0 \pm 0.8$ & $5.9 \pm 0.7$ & $3.1 \pm 0.3$ & $5.5 \pm 0.7$ \\
$\begin{array}{c}\text { Kijko-Sellevoll- } \\
\text { Bayes }\end{array}$ & $5.6 \pm 0.4$ & $5.6 \pm 0.4$ & $3.0 \pm 0.2$ & $5.1 \pm 0.3$ \\
$\begin{array}{c}\text { Robson- } \\
\text { Whitlock }\end{array}$ & $5.6 \pm 0.5$ & $6.1 \pm 0.9$ & $3.1 \pm 0.3$ & $5.3 \pm 0.5$ \\
$\begin{array}{c}\text { Robson- } \\
\text { Whitlock-Cooke }\end{array}$ & $5.4 \pm 0.2$ & $5.6 \pm 0.5$ & $3.0 \pm 0.2$ & $5.0 \pm 0.3$ \\
$\begin{array}{c}\text { Mmax Estimada } \\
\text { (Promedio) }\end{array}$ & 5.6 & 5.8 & 3.0 & 5.2 \\
& & & & $5.4 \pm 0.2$ \\
& & & $5.4 \pm 0.6$ \\
& & &
\end{tabular}

\section{Líneas futuras y próximos pasos a realizar.}

Tras este primer análisis realizado de la serie sísmica entorno al embalse de Pirrís, las próximas tareas a realizar están dirigidas a trabajar en los aspectos más físicos ligados a la ocurrencia de sismos asociados al llenado de embalses, ahondando en el estudio de los cambios de esfuerzos en la zona que puedan influir en la ocurrencia de nuevos sismos. Con la serie sísmica actualizada y relocalizada hasta finales de 2016 se calcularán, en primer lugar, los cambios de esfuerzos estáticos de Coulomb debido al peso vertical de la columna de agua en el embalse. Posteriormente se analizarán posibles cambios de esfuerzos poro elásticos debidos a infiltraciones de agua, estudiando el proceso de difusión de presión de poros por el medio. Todo esto permitirá definir una función de densidad de magnitudes que será un input de entrada fundamental para la metodología que se desea desarrollar, dirigida a la estimación de la peligrosidad sísmica incluyendo sismicidad inducida o sismicidad disparada.

Además se quiere probar si la función de magnitudes que finalmente se obtenga se ajusta a otros casos de llenado de embalses y si podrá ser utilizada para predecir de cierta manera la sismicidad inducida y/o disparada a corto-medio plazo en otras obras hidroeléctricas, como es el caso del C.P. de Reventazón (Costa Rica).

\section{Conclusiones}

El análisis hasta ahora realizado, ha permitido encontrar diferentes indicadores que permiten confirmar la existencia de sismicidad asociada al llenado del embalse. Lo que no es tan claro es la correlación con los cambios en el nivel del embalse en los sucesivos años y la sismicidad. Algunos de estos indicadores son:

- Aumento de la sismicidad en los alrededores del embalse tras el inicio de la operación de llenado, lo que se refleja en la tasa diaria de sismos en las áreas analizadas alrededor del mismo (5 y 20 km)

- Se comprueba como la sismicidad cambia espacialmente antes y después del llenado, aumentándose la concentración de sismos en las proximidades del Norte del embalse.

- El valor del parámetro b es próximo a 2.0 tras la operación de llenado.

- Los cambios temporales del valor de b podrían estar indicando la presencia de un fenómeno de difusión de la presión de poros.

- Siguiendo la clasificación de sismicidad disparada en función de la magnitud, propuesta por Guha and Patil (1990), en el CP de Pirrís estaríamos ante un caso de microsismicidad disparada durante el llenado y de una sismicidad moderada-débil tras el llenado.

- Aplicando diferentes técnicas (Kijko 2004) las estimaciones de magnitud máxima Mmax llegan a valores de 5,8, que son mayores que la Mmax observada de 5,2.

- Aunque existen fallas locales cerca del embalse, que por sus condiciones podrían ser reactivadas, son fallas pequeñas con un potencial para generar sismos de magnitudes moderadas (Mmax 5,8). Sin embargo, en los alrededores existen fallas de mayor tamaño.

\section{Agradecimientos}

Agradecimiento especial al Instituto Costarricense de Electricidad, por facilitarnos el acceso a toda su información para la realización de este estudio.

\section{References}

BARQUERO, R. and ALVARADO, G., 2012. Actividad sísmica 2008-2009 en los alrededores del Centro de Producción de Pirrís, Informe Interno ICE.

BARQUERO, R. and CLIMENT, A., 2006. Estudio sismológico y tectónico para la cuenca del río Pirrís, Informe Interno ICE.

BARQUERO, R., LÓPEZ, A. and BARRANTES, J., 2001. Proyecto hidroeléctrico Pirrís: Estudio del potencial de sismicidad inducida. Inf. Interno ICE. $71 \mathrm{pp}$. 
BOURNE, S.J., OATES, S.J., BOMMER, J.J., DOST, B., VAN ELK, J. and DOORNHOF, D. 2015. A Monte Carlo Method for Probabilistic Hazaard Assessment of Induced Seismicity due to Conventional Natural Gas Production. Bull. Seismol. Soc. Am. 105, pp. 1721-1738.

CLIMENT, A. and PIEDRA, R. 2012. Instrumentación acelerográfica presa C.P. Pirrís. Análisis de los registros acelerométricos obtenidos en la presa Pirrís ante eventos sísmicos ocurridos en el 2011 y 2012 . Informe interno Instituto Costarricense de Electricidad.

CONVERTITO V., MAERCKLIN N., SHARMA N., and ZOLLO A., 2012. From Induced Seismicity to Direct TimeDependent Seismic Hazard. Bull. Seismol. Soc. Am. 102, no. 6, pp. 2563-2573.

DENYER, P., MONTERO, W. and ALVARADO, G.E., 2003. Atlas tectónico de Costa Rica. 81 pp. Edit. Univ. de Costa Rica, San José.

Embalses de Plantas Hidroeléctricas ICE, Informe interno área de Ingeniería Hidráulica (ICE), agosto 2012.

GUHA, S.K and PATIL, D.N., 1990. Large water reservoir related induced seismicity, Gerlands Beitr. Geophysik, 99, pp. 3-5.

GUPTA, H.K. 1983. Induced seismicity hazard mitigation through water level manipulation at Koyna, India: A suggestion, Bull. Seism. Soc. Am., 73, pp. 679-682.

GUPTA, H.K., 2002. A review of recent studies of triggered earthquakes by artificial water reservoirs with special emphasis on earthquakes in Koyna, India. Earth-Science Reviews 58, pp. 279-310.

GUPTA, H.K., MANDAL, B.K. and RASTOGI, 2002. How long will triggered earthquakes at Koyna, India, continue?, Current Science, 2, pp. 202-210.

GUPTA, H.K., RASTOGI, B.K. and NARAIN, H., 1972a. Common features of the reservoir associated seismic activities. Bull. Seismol. Soc. Am. 62, pp. 481- 492.

GUPTA, H.K., RASTOGI, B.K. and NARAIN, H., 1972b. Some discriminatory characteristics of earthquakes near the Kariba, Kremasta and Koyna artificial lakes. Bull. Seismol. Soc. Am. 62, pp. 493-507.

GUTENBERG, B. and RICHTER, C.F., 1944. Frequency of earthquakes in California. Bulletin of the Seismological Society of America. 34, pp. 185-188.

ICE, 2011. Informe geológico viviendas dañadas barrio las Rojas-Zapotal (2011), ICE.

ICE, 2006. Informe Interno ICE. Red sismológica para el P.H. Pirris.

ICOLD, International Commission On Large Dams, 2004. Committee on Seismic Aspects of Dam Design. Reservoir and seismicity. State of Knowledge.

KEBEASY, R. M., MAAMOUN, M., IBRAHIM, E., MEGAHED, A., SIMPSON, D. W. and LEITH, W. S., 1997. Earthquake studies at Aswan reservoir, J. Geodynam., 7, 173-193.

KIJKO, A., 2004. Estimatiom of the maximum earthquake magnitude, mmax, Pure and Applied Geophysics 161(8), pp. 1655-1681.

KIJKO, A. and SINGH, M., 2011. Statistical Tools for Maximum Possible Earthquake Magnitude Estimation. Acta Geophysica, 59, 4, pp. 674-700. DOI:10.2478/s11600-011-0012-6.

MCGARR, A. and SIMPSON, D., 1997. In "Rockbursts and Seismicity in Mines", pp. 385-396, Balkema.

MONTERO, W. y CLIMENT, A. 1990. Los terremotos de Grecia de 1882 y de Quepos de 1952, Costa Rica, distribución de intensidades y origen tectónico. Ciencia y Tecnología, 14(1-2), pp. 81-105. San José, Costa Rica.

MORA, S., VALVERDE, R. and BRENES, G., 1985. Análisis Geológico-Geomorfológico de la Cuenca del río Pirrís (Parrita) Costa Rica. Estudio del Dpto Ingeniería Forestal ITCR, Cartago Costa Rica.

PETERSEN, M.D., MUELLER, C.S., MOSCHETTI, M.P., HOOVER, S.M., SHUMWAY, A.M., MCNAMARA, D.E., and RUBINSTEIN, J.L., 2017. 2017 one-year seismic-hazard forecast for the Central and Eastern United States from induced and natural earthquakes. Seismological Research Letters, 88(3), pp. 772-783.

RED SISMOLÓGICA NACIONAL (RSN: ICE-UCR): Banco de datos sismológicos de Costa Rica.

ROJAS, W., 1993. Catálogo de sismicidad histórica y reciente en América Central: Desarrollo y análisis. Tesis de Licenciatura. Norwegian Seismic Array (NORSAR), Noruega.

SIMPSON, D.W., LEITH, W.S. and SCHOLZ, C.H., 1988. Two types of reservoir-induced seismicity. Bulletin of the Seismological Society of America, 78(6), pp. 2025-2040.

TALWANI, P., 1987. On nature of reservoir-induced seismicity. Pure Appl. Geophys. 150, pp. 473-492.

WELLS, D.L., and COPPERSMITH, K. J., 1994. New Empirical Relationships among Magnitude, Rupture Length, Rupture Width, Rupture Area and Surface Displacement. Bulletin Seismological Society of America, Vol. 84, No. 4. 